\title{
Elongated Photonic Nanojet from Truncated Cylindrical Zone Plate
}

\author{
Sergey S. Stafeev and Victor V. Kotlyar \\ Laser Measurements Laboratory, Image Processing Systems Institute, Russian Academy of Sciences, \\ 151 Molodogvardeyskaya Street, Samara 443001, Russia \\ Correspondence should be addressed to Sergey S. Stafeev, sergey.stafeev@gmail.com
}

Received 31 July 2012; Accepted 18 October 2012

Academic Editor: Keli Han

Copyright ( 12012 S. S. Stafeev and V. V. Kotlyar. This is an open access article distributed under the Creative Commons Attribution License, which permits unrestricted use, distribution, and reproduction in any medium, provided the original work is properly cited.

Previously (Chen et al., 2004), it was shown that dielectric cylinder can form focal spots with small diameters and long depth. This type of focal spot was called photonic nanojet. In this paper, it was shown that dielectric cylinder of radius $595 \mathrm{~nm}$ (1.12 of wavelength) forms near the surface a photonic nanojet with diameter equal to 0.31 of wavelength and depth of focus equal to 0.57 of wavelength. Adding truncated concentric rings with radiuses equal to radiuses of zone plate to the cylinder increases the depth of focus to 1.18 of the wavelength. The diameter and intensity of focal spot near the cylinder surface remain unchanged.

\section{Introduction}

One of the promising areas of research in nanophotonics is tight focus that is formed using dielectric cylinders or spheres with diameters about wavelength. This type of focal spot was called "photonic nanojet" [1]. Unique features of photonic nanojets are small diameter and low divergence of the beam.

The name "photonic nanojet" was for the first time used in [1]. In [2], photonic nanojet was formed using dielectric spheres with different diameters illuminated by plane wave $(\lambda=400 \mathrm{~nm})$. Also in [2], it was shown that the spheres with diameters of $1 \mu \mathrm{m}$ and $2 \mu \mathrm{m}$ formed photonic nanojets with diameters in the plane perpendicular to the direction of incident polarization equal to $0.325 \lambda$ and $0.375 \lambda$. In [3], photonic nanojet with the maximum intensity 150 times larger than the intensity of the incident beam was obtained. In [4] photonic nanojet was obtained experimentally using scanning confocal microscope. It was shown that latex sphere with diameter equals to $3 \mu \mathrm{m}$ forms focal spot with a diameter $\mathrm{FWHM}=270 \mathrm{~nm}$ (the wavelength equals to $520 \mathrm{~nm}$ ). The influence of incident light polarization was investigated in [5]. It was shown that radially polarized light formed photonic nanojet with effective focal volume 10 times smaller than the circularly polarized light. Photonic nanojet could be formed not only by spheres but by finite or infinite cylinders. For instance, in [6] photonic nanojet formed by discs from silica nitride with width $400 \mathrm{~nm}$ and diameters from 1 to $10 \mu \mathrm{m}$ was obtained experimentally. The diffraction of light by multilayers cylinders was considered in [7]. Dielectric sphere with properly graded refractive index was used in [8] to increase the length of photonic nanojet. The length of a photonic nanojet formed by the gradedindex microsphere can be increased to approximately 20 wavelengths.

\section{Numerical Modeling}

The diffraction of the TE-polarized plane wave was simulated using FDTD method [9] implemented in FullWAVE [10]. The mesh grid size in space was equal to $0.01 \mu \mathrm{m}(0.02$ of wavelength), in time $-0.007 \mu \mathrm{m} / c$, where $c$ is the velocity of light. Perfectly matched layers with width of $0.5 \mu \mathrm{m}$ were located on the borders of calculated area. TE-polarized plane wave $(\lambda=532 \mathrm{~nm})$ was used as the incident wave.

\section{Cylinder}

First, we numerically investigate propagation of TE-polarized light throw 2D dielectric cylinder with radius $0.595 \mu \mathrm{m}$ and refractive index $n=1.5$. Figure 1 depicts intensity 


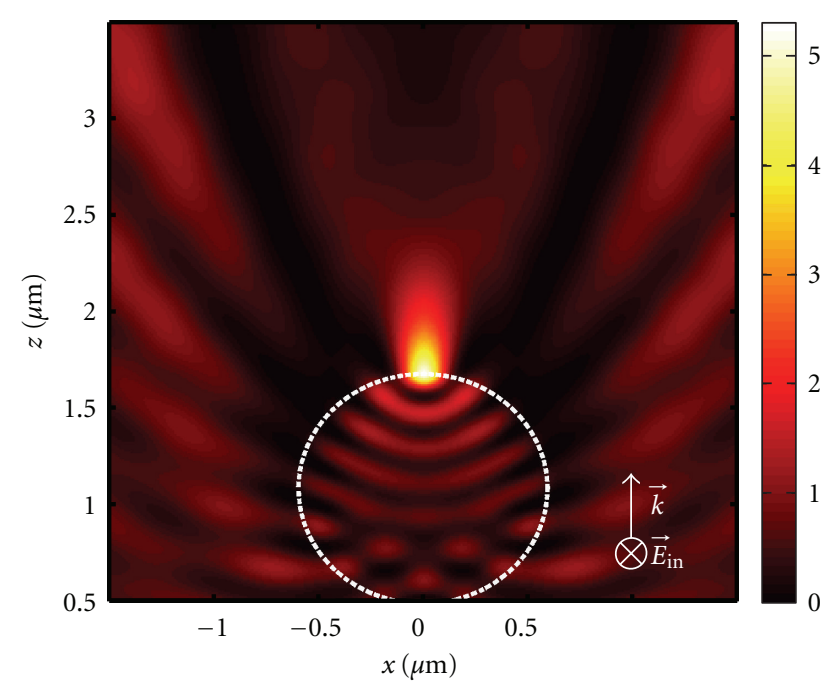

FIgURE 1: Intensity distribution in fragment of the calculated area for the dielectric cylinder with radius $0.595 \mu \mathrm{m}$. White dashed line shows the cylinder border.

distribution in calculated area. Intensity distribution along $z$-axis is shown in Figure 2. From Figure 2 the depth of focus equals to $\mathrm{DOF}=0.57 \lambda$. Intensity distribution in focal plane is shown in Figure 3. Diameter of focal spot near the surface equals to FWHM $=0.31 \lambda$. On the distance of $0.1 \mu \mathrm{m}$ from cylinder surface, the diameter of focal spot equals to $\mathrm{FWHM}=0.38 \lambda$. Both diameters are smaller than the diffraction limit for $2 \mathrm{D}$ case $(\mathrm{FWHM}=0.44 \lambda)$.

\section{Truncated Cylindrical Zone Plate}

To increase the depth of focus formed by dielectric cylinder, we used nonclosed concentric dielectric rings with refractive index $n=1.5$. Radiuses of the rings were calculated using equation for Fresnel zone plate $r_{m}{ }^{2}=\lambda m f+\lambda^{2} m^{2} / 4$, where $f=532 \mathrm{~nm}$. 12 rings were added to the cylinder. Thus TE-polarized wave was focused by $2 \mathrm{D}$ truncated zone plate. Figure 4 depicts intensity distribution in calculated area. Intensity distribution along $z$-axis is shown in Figure 5. Figure 5 depicts that there are two peaks of intensity along $z$-axis. Diameter of focal spot near the surface is equal to $\mathrm{FWHM}=0.30 \lambda$ (Figure 6). But diameter of focal spot formed by truncated zone plate increases more rapidly than the focal spot formed by cylinder. On the distance of $0.1 \mu \mathrm{m}$ from cylinder surface, the diameter of focal spot is equal to FWHM $=0.40 \lambda$. The depth of focus is equal to DOF $=$ $1.18 \lambda$ and is two times larger than the depth of focus formed by cylinder. So adding of nonclosed concentric rings to the cylinder does not change the diameter of focal spot near the surface but increases the depth and divergence of the beam.

\section{Conclusion}

In this paper, it was shown that $2 \mathrm{D}$ dielectric cylinder $(n=$ 1.5) with radius $1.12 \lambda$ illuminated by TE-polarized plane wave $(\lambda=532 \mathrm{~nm})$ forms photonic nanojet with diameter

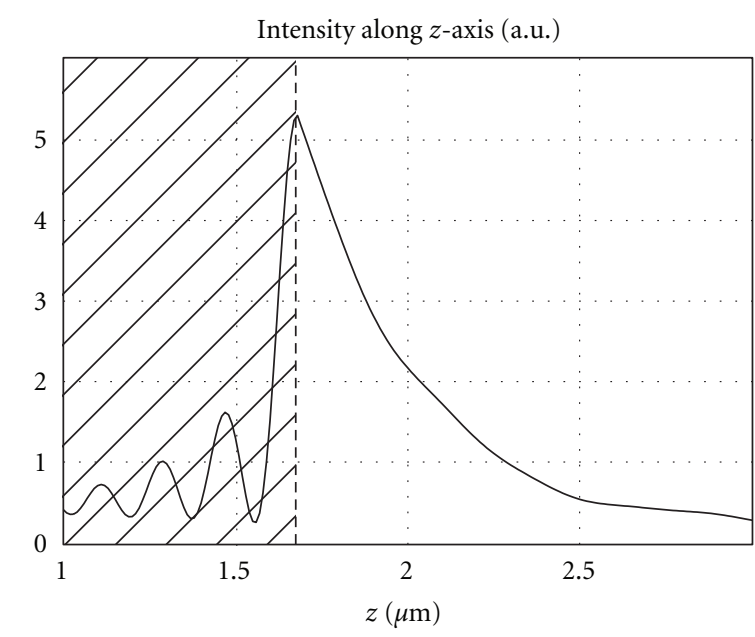

FIGURE 2: Intensity distribution along $z$-axis for the dielectric cylinder with radius $0.595 \mu \mathrm{m}$.

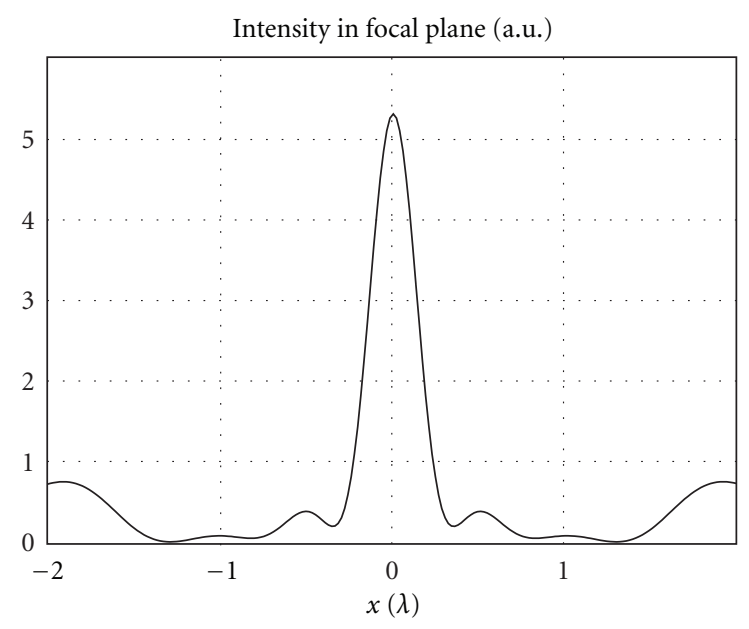

FIgURe 3: Intensity distribution in focal plane for the dielectric cylinder with radius $0.595 \mu \mathrm{m}$.

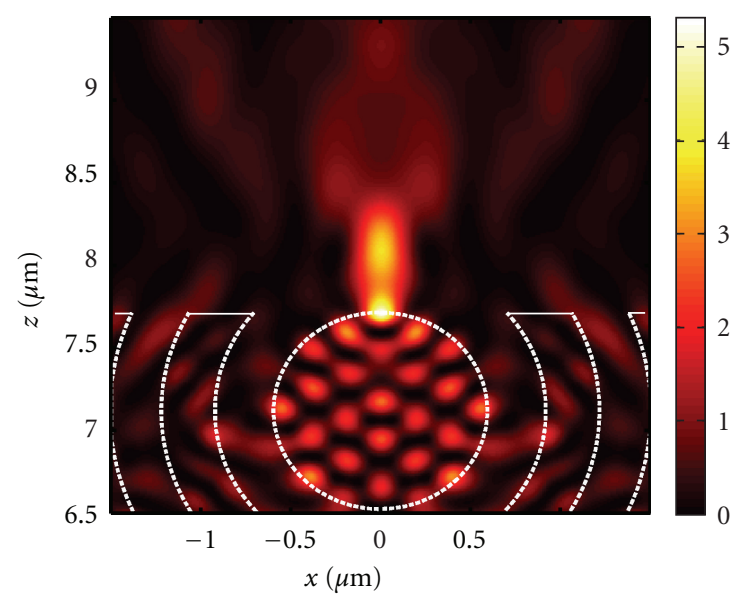

FIGURE 4: Intensity distribution in fragment of the calculated area for the truncated zone plate. White dashed line shows the cylinder border. 


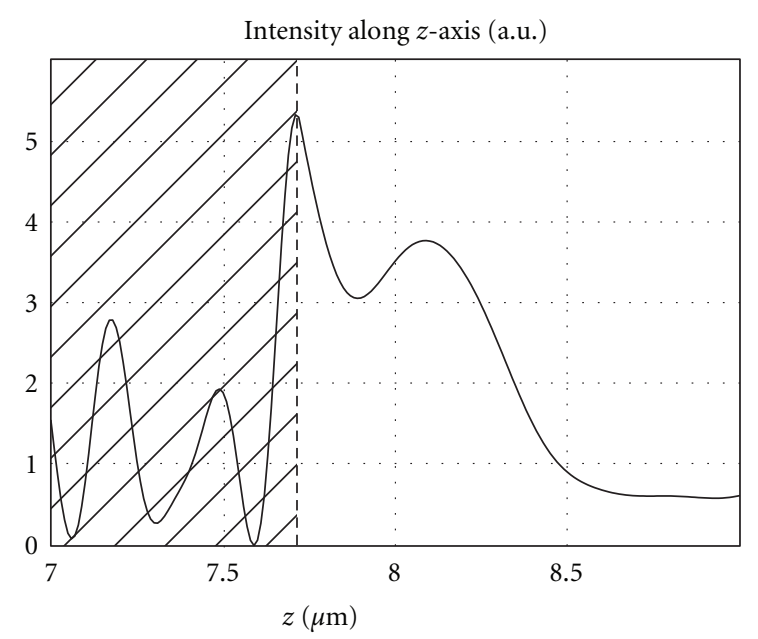

FIGURE 5: Intensity distribution along $z$-axis for the truncated zone plate.

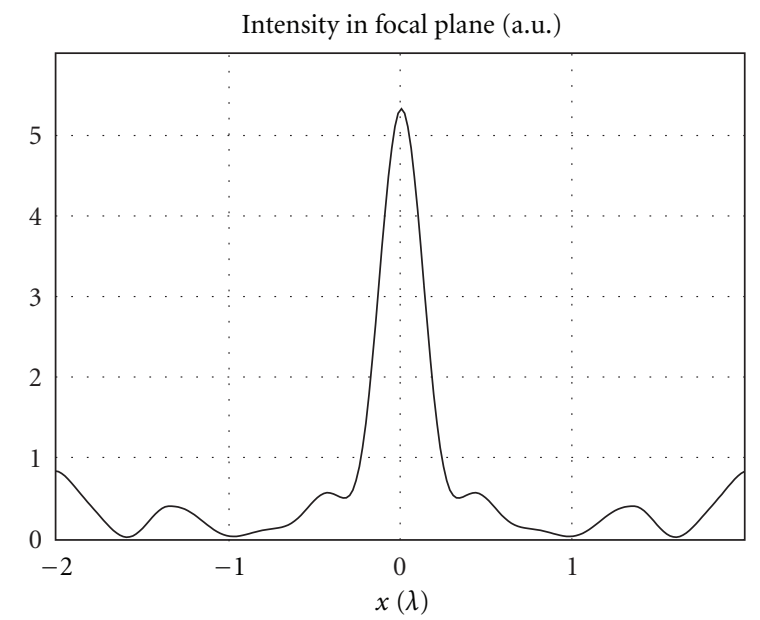

Figure 6: Intensity in focal plane for the truncated zone plate.

$0.31 \lambda$ and depth $0.57 \lambda$. Adding truncated concentric rings with radiuses equal to radiuses of zone plate to the cylinder increases the depth of focus to $1.18 \lambda$. The diameter and intensity of focal spot near the cylinder surface remain unchanged.

\section{Acknowledgments}

The work was financially supported by the Federal Program "Research and Educational Staff of Innovation Russia" (State Contract no. 14.740.11.0016), Russian Federation Presidential Grants for Support of Leading Scientific Schools (NSh-4128.2012.9) and a Young Researcher's Grant (MK3912.2012.2), and the Russian Foundation for Basic Research Grant (12-07-00269, 12-07-31115, 12-07-31117).

\section{References}

[1] Z. Chen, A. Taflove, and V. Backman, "Photonic nanojet enhancement of backscattering of light by nanoparticles: a potential novel visible-light ultramicroscopy technique," Optics Express, vol. 12, no. 7, pp. 1214-1220, 2004.
[2] X. Li, Z. Chen, A. Taflove, and V. Backman, "Optical analysis of nanoparticles via enhanced backscattering facilitated by 3-D photonic nanojets," Optics Express, vol. 13, no. 2, pp. 526-533, 2005.

[3] S. Lecler, Y. Takakura, and P. Meyrueis, "Properties of a threedimensional photonic jet," Optics Letters, vol. 30, no. 19, pp. 2641-2643, 2005.

[4] P. Ferrand, J. Wenger, A. Devilez et al., "Direct imaging of photonic nanojets," Optics Express, vol. 16, no. 10, pp. 6930-6940, 2008.

[5] Y. Liu, B. Wang, and Z. Ding, "Influence of incident light polarization on photonic nanojet," Chinese Optics Letters, vol. 9, no. 7, Article ID 072901, 2011.

[6] D. McCloskey, J. J. Wang, and J. F. Donegan, "Low divergence photonic nanojets from $\mathrm{Si}_{3} \mathrm{~N}_{4}$ microdisks," Optics Express, vol. 20, pp. 128-140, 2012.

[7] V. V. Kotlyar and M. A. Lichmanov, "Analysis of electromagnetic wave diffraction on an infinite circular cylinder with several layers of homogeneity," Computer Optics, vol. 24, pp. 26-32, 2002 (Russian).

[8] S.-C. Kong, A. Taflove, and V. Backman, "Quasi one-dimensional light beam generated by a graded-index microsphere," Optics Express, vol. 17, no. 5, pp. 3722-3731, 2009.

[9] A. Taflove and S. C. Hagness, Computational Electrodynamics: The Finite-Difference Time-Domain Method, Artech, Norwood, Mass, USA, 3rd edition, 2005.

[10] http://www.rsoftdesign.com/products.php?sub=Component + Design\&itm=FullWAVE . 

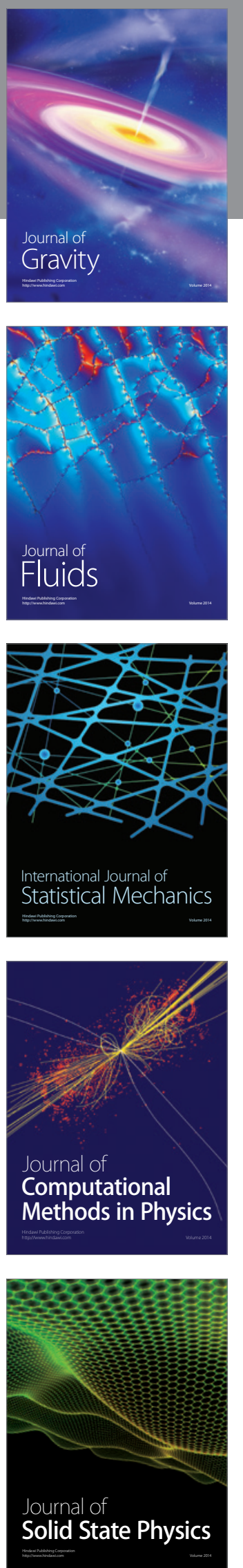

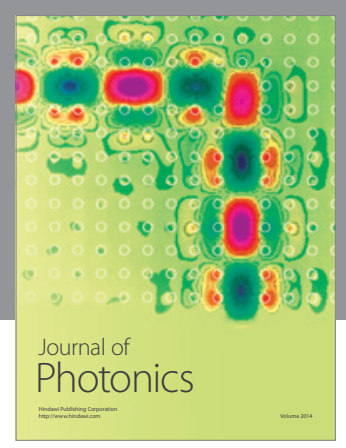

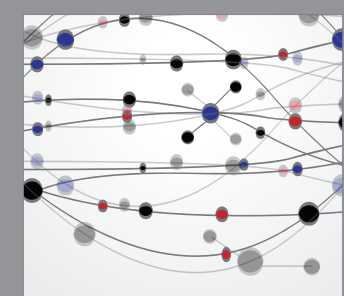

The Scientific World Journal
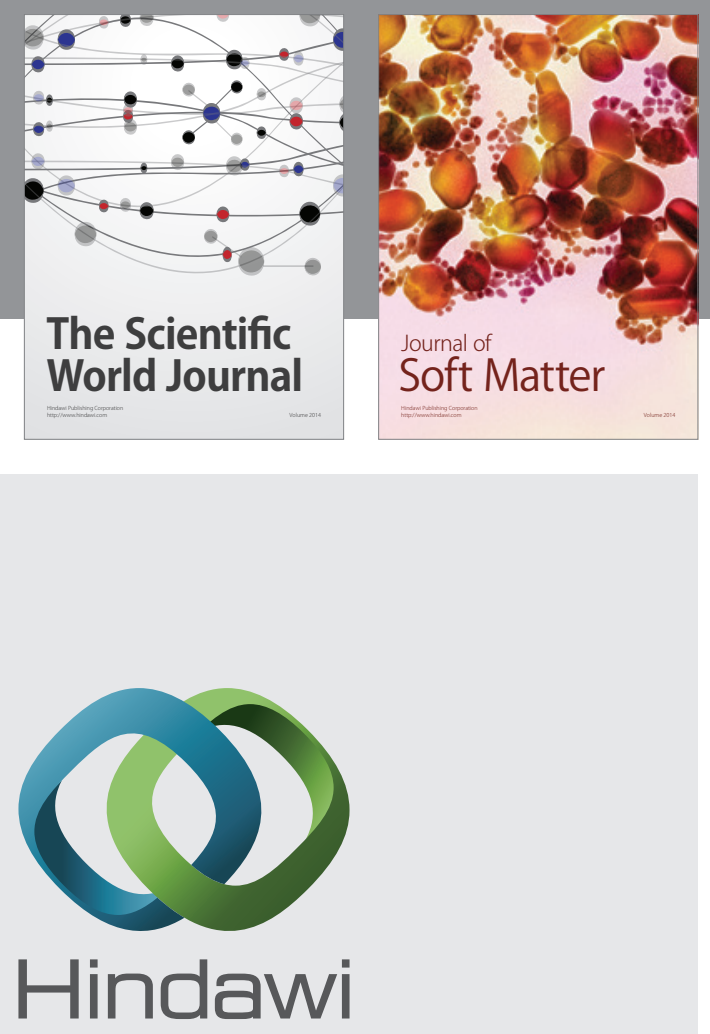

Submit your manuscripts at

http://www.hindawi.com
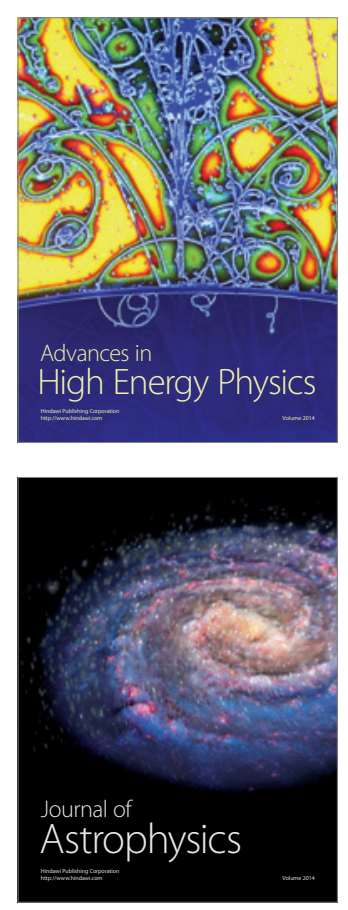
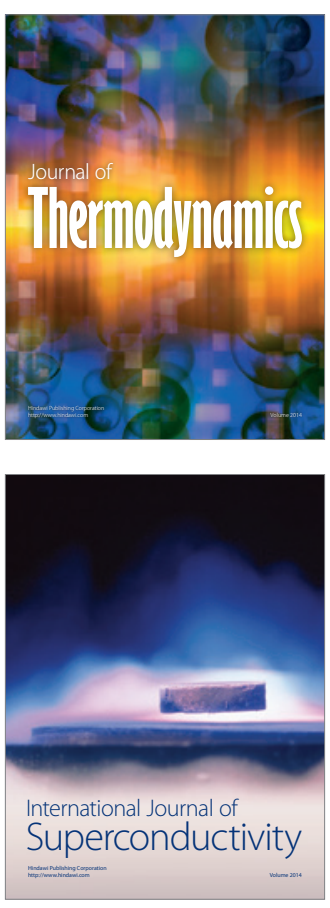
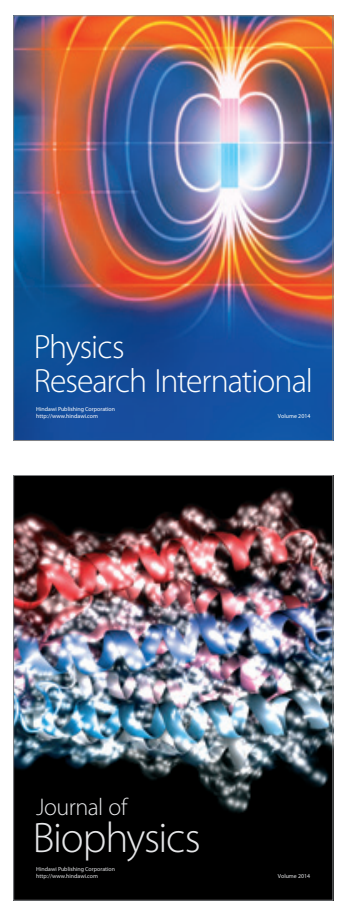
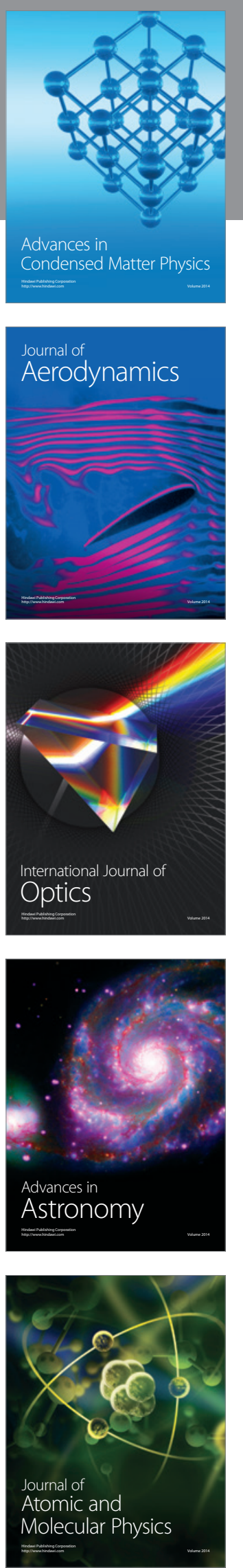